\title{
Simulation of Flow in Hydraulic Structures using ADH
}

\section{US Army Corps of Engineers}

\author{
by R. L. Stockstill and R. C. Berger
}

PURPOSE: The purpose of this Technical Note is to describe a process of modeling flow in hydraulic structures. In particular, application is made with the unstructured flow solver, ADH (ADaptive Hydraulics model). ADH also contains solvers for groundwater and two-dimensional shallow water flows. It is capable of refining or coarsening the grid based on error estimates during flow calculations. Three demonstration applications are presented: (a) CAD-to-Grid Process Demonstration at an Intake, (b) Scalability Demonstration with Outlet Manifold, and (c) Free-surface Capability Demonstration with Supercritical Flow Contraction.

INTRODUCTION: Modeling of hydraulic structures presents many computational challenges to design/evaluation engineers. Hydraulic structures often have components containing internal (pressure) flow while other components hold external (free-surface) flow. Therefore, threedimensional (nonhydrostatic) codes must account for both regions of fixed domain limits and regions that have time-varying domains because of free surface movement. Multiple regions of rapid accelerations also characterize hydraulic structures as water passes from slow reservoir flow to high-velocity conduit flow. This difference translates to significant differences in Reynolds number over various portions of the modeled flow field. Free-surface applications to steep channels such as chutes require a model that produces stable solutions for a large range of Froude numbers. The model must solve subcritical and supercritical flow fields and resolve any transitions between these regimes. These flow fields may contain shocks such as oblique standing waves and hydraulic jumps. The solutions may include a highly contorted free surface, and thus requires a robust free-surface model. Finally, the geometric complexity of most hydraulic structures necessitates the use of CAD (Computer Aided Design/Drafting) modeling which leads to a need for a CAD-to-grid generator interface. Visualization, of course, is also important for the engineer to examine the geometric design and the resulting hydraulic conditions.

MODELING PROCEDURE: The process of developing computational models of hydraulic structures begins with a three-dimensional CAD description of the parameter-based geometry. This CAD information is then fed to a grid generator where the domain is discretized into a tetrahedral mesh required by ADH (Berger and Stockstill 1999). The resulting mesh and specified boundary conditions are then passed as input to the flow solver. The resulting solution must next be transferred to a visualization tool to facilitate the evaluation of the hydraulic conditions within the structure. The flow solver is capable of determining the internal pressure and elevation of the free surface along with the three velocity components without assuming a hydrostatic pressure distribution. The ADH code is written to take advantage of the newest multiple-processor machines of the Major Shared Resource Center (MSRC), Engineer Research and Development Center (ERDC) Information Technology Laboratory (ITL). 
APPLICATIONS: Application of ADH has been made to hydraulic structures varying from high-velocity channels to navigation components. This technical note presents three applications as demonstrations of the modeling process, and it documents the model's features and capabilities. In each case the flow solution was run for steady-state conditions.

CAD-to-Grid Process Applied to an Intake: The first application is a model of the Marmet Navigation Lock intake structure. The multiported intake model demonstrates the required CAD-to-Grid process for complicated geometries. The intake modeled about $215 \mathrm{~m}$ of the upper approach, the upper miter gates and recesses, the intake ports, and filling system culverts as shown in the CAD drawing provided in Figure 1. The tetrahedral mesh, shown in Figure 2, had 20,194 nodes and 87,953 elements. Figure 3 shows the details of the discretization near the intake ports. Flow in the approach channel is slow and deep. The flow accelerates as it enters the intake ports. Further accelerations occur as the port flows combine into a single culvert on each half of the structure. These accelerations are shown in the velocity and pressure contours along a plane located in the center of the culvert beginning at the port faces (Figures 4 and 5, respectively).

Scalability Demonstrated with Outlet Manifold: Next, the parallel performance of the ADH code is shown by application of the flow model to a multiported navigation lock outlet. The outlet model is an application requiring high resolution to describe the port shapes as shown on the surface mesh of Figure 6. The tetrahedral mesh had 48,000 nodes and 244,000 elements. Initial runs were made to obtain computational timing information for documentation of ADH's scalability. The model was run on a Department of Defense (DoD) High-Performance Computing Center's Cray T3E. The multiple processor speedups are illustrated by the timings presented in Figure 7. Significant speedups were obtained when up to 64 processors were used. Additional processors did not speed up the computation time in this application due to the larger relative communication time required between processors. This is a relatively small test case. Parallel performance will be even better for larger problems. The flow solution is shown on the velocity contours plotted on a horizontal plane through the center of the structure (Figure 8).

\section{Free-surface Capability Demonstrated with Supercritical Flow Contraction:}

Finally, free-surface capabilities are demonstrated by application to a supercritical flow contraction having an approach Froude number of 4. This application demonstrates ADH's freesurface modeling capabilities in flow fields having multiple shocks. The free-surface solution is challenging due to the presence of oblique standing waves within and downstream of the transition. The flow solution is presented on the steady-state finite element mesh shown on Figure 9. The top of the mesh maps the oblique standing waves generated within the contraction.

SUMMARY AND CONCLUSIONS: The ADH flow solver will provide a rapid and economical method of predicting the hydrodynamic conditions in and around hydraulic structures. The parallel code allows reasonable computational time requirements for extremely large problems.

RECOMMENDATIONS FOR FUTURE RESEARCH: Accurate modeling of hydraulic structures requires accounting for turbulence effects. Shear stresses generated at fixed 
boundaries play an important role in the composition of flow in conduits and channels. Future efforts should include the development of a turbulence model within ADH.

ADDITIONAL INFORMATION: Funding for this effort was provided by the U.S. Department of Defense High Performance Computing Modernization Office through the Common Highperformance Software Support Initiative (CHSSI). Additional information may be obtained from Dr. R. L. Stockstill, Coastal and Hydraulics Laboratory, U.S. Army Engineer Research and Development Center (ERDC) at 601-634-4251 or e-mail

Richard.L.Stockstill@erdc.usace.army.mil; or Dr. R. C. Berger, Coastal and Hydraulics

Laboratory, U.S. Army Engineer Research and Development Center (ERDC) at 601-634-2570 or e-mail Charlie.R.Berger@erdc.usace.army.mil.

\section{REFERENCE:}

Berger, R. C., and Stockstill, R. L. (1999). "A finite element system for flows," Proceedings of the 1999 ASCE Water Resources Engineering Conference, "Water Resources into the New Millenium, Past Accomplishments and New Challenges," Seattle, WA.

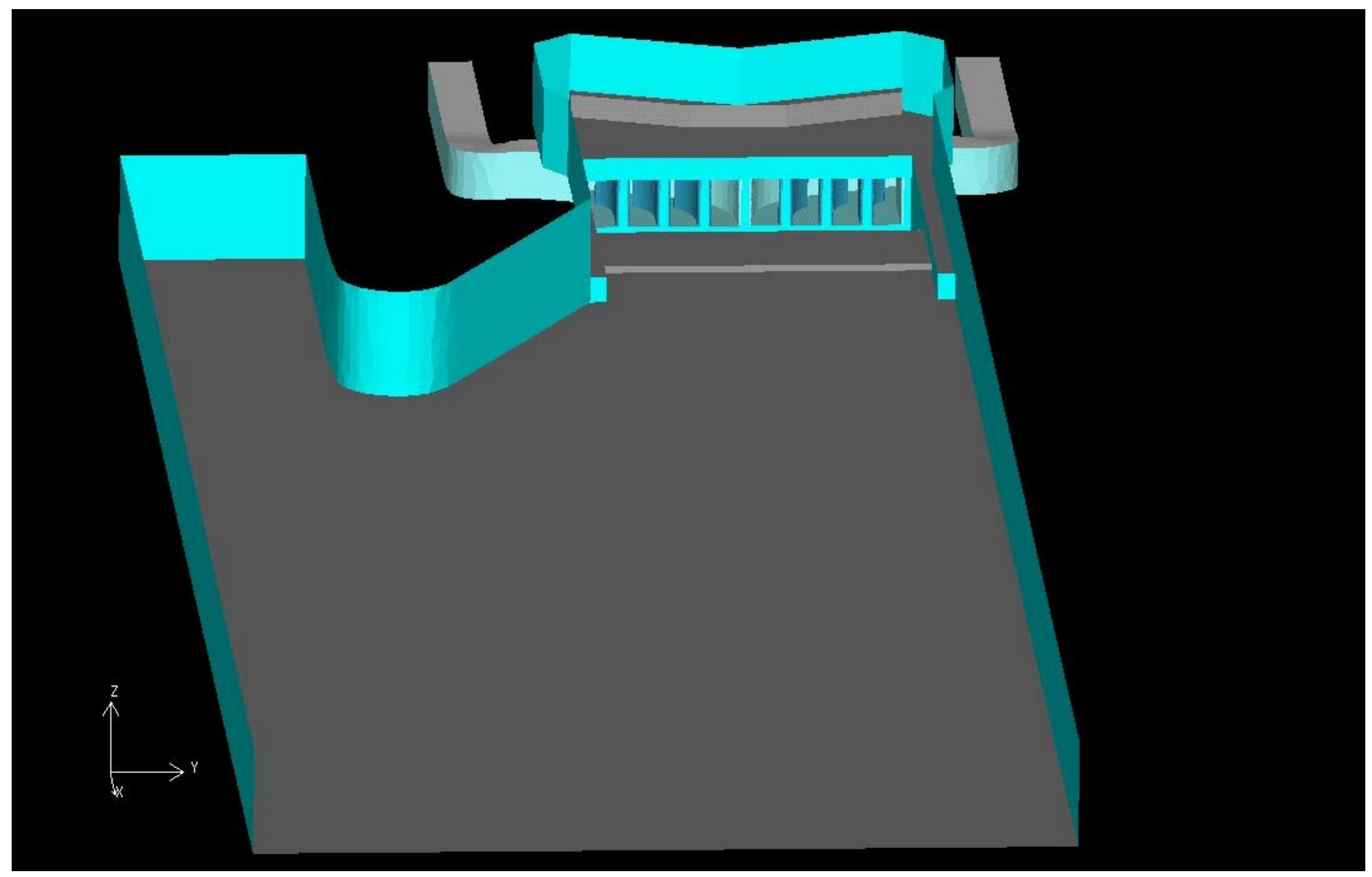

Figure 1. Three-dimensional CAD model of lock intake 


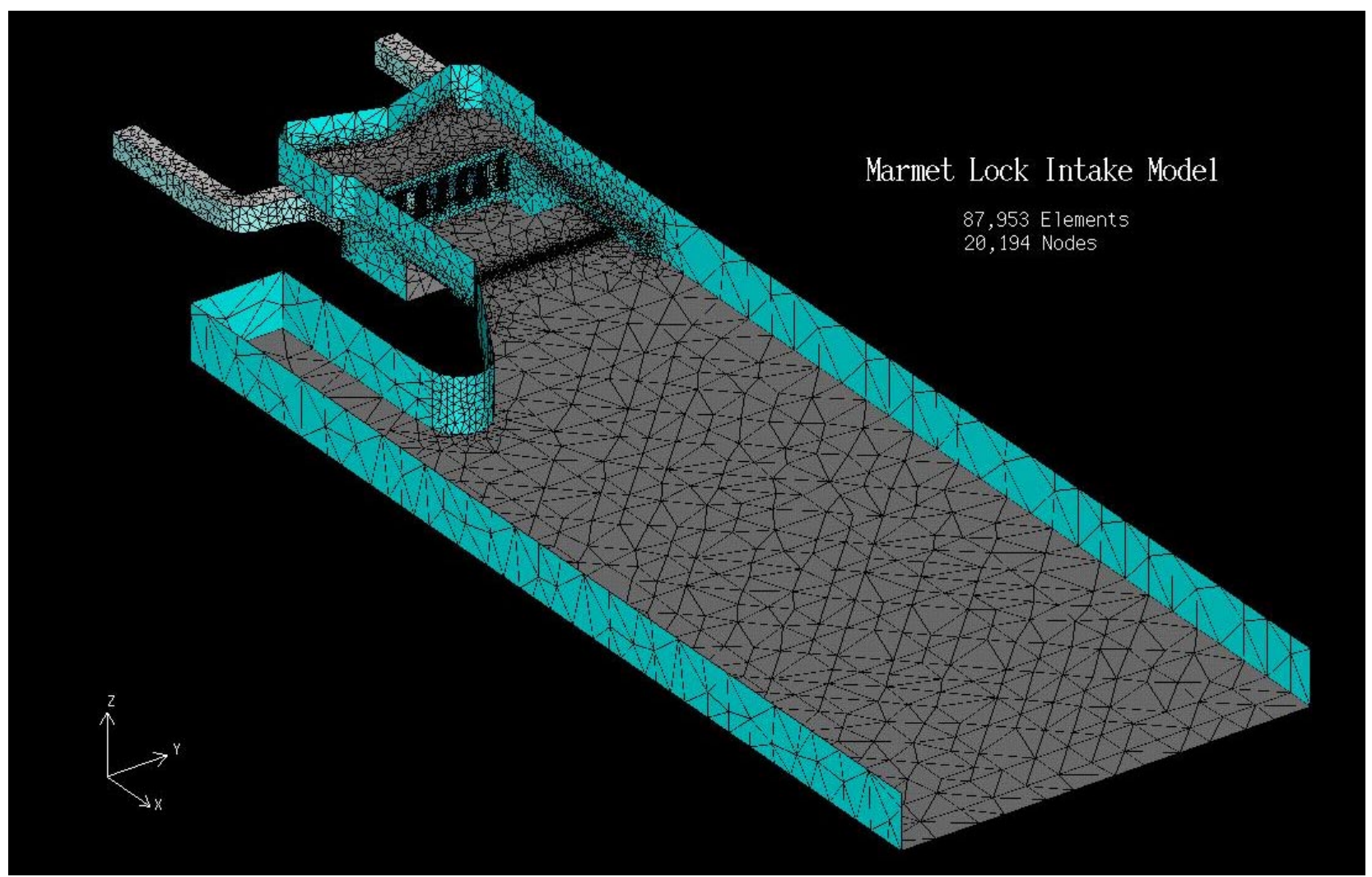

Figure 2. Surface mesh of lock intake

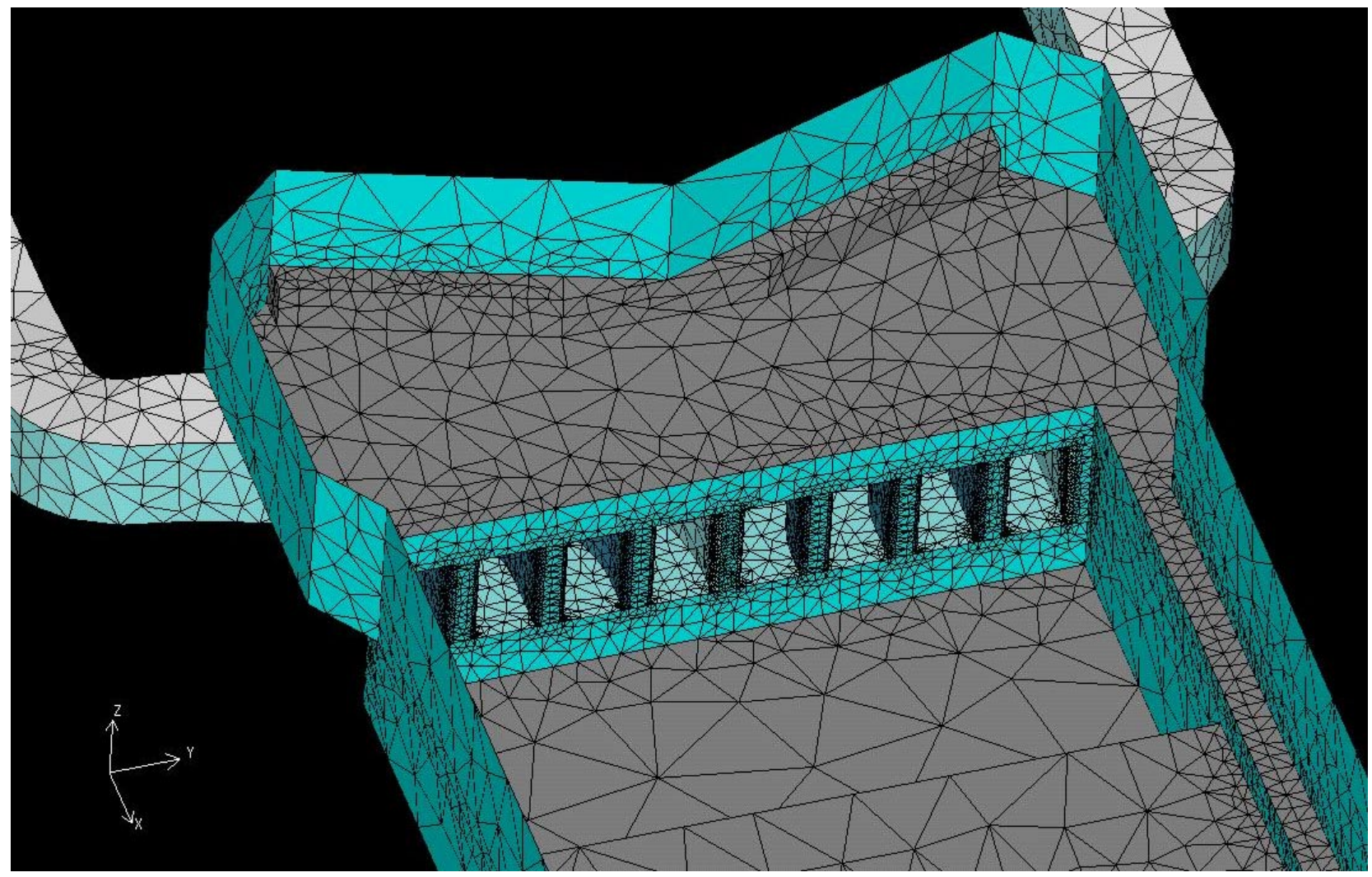

Figure 3. Surface model at the port faces 


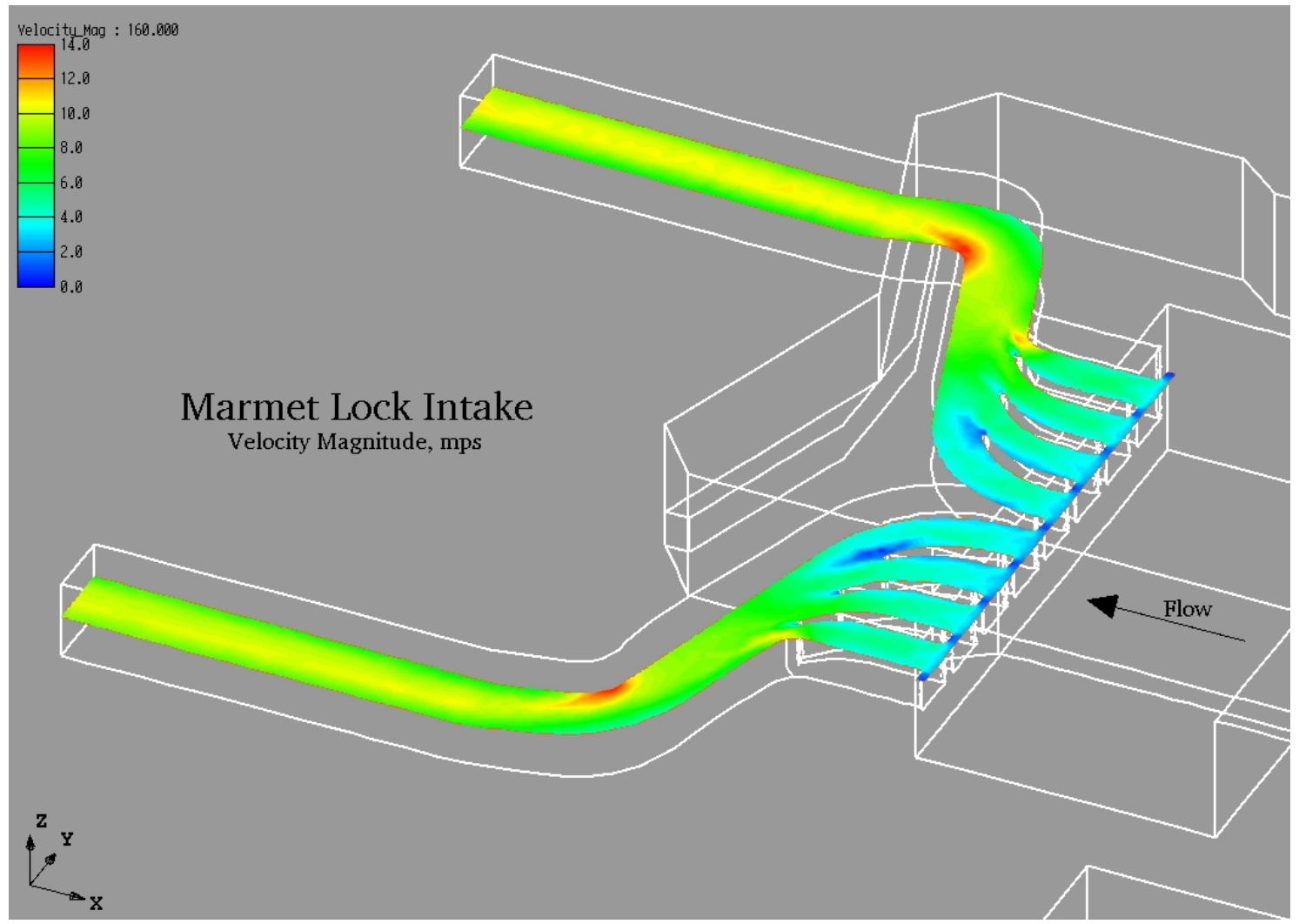

Figure 4. Velocity contours on a plane through center of intake and culverts

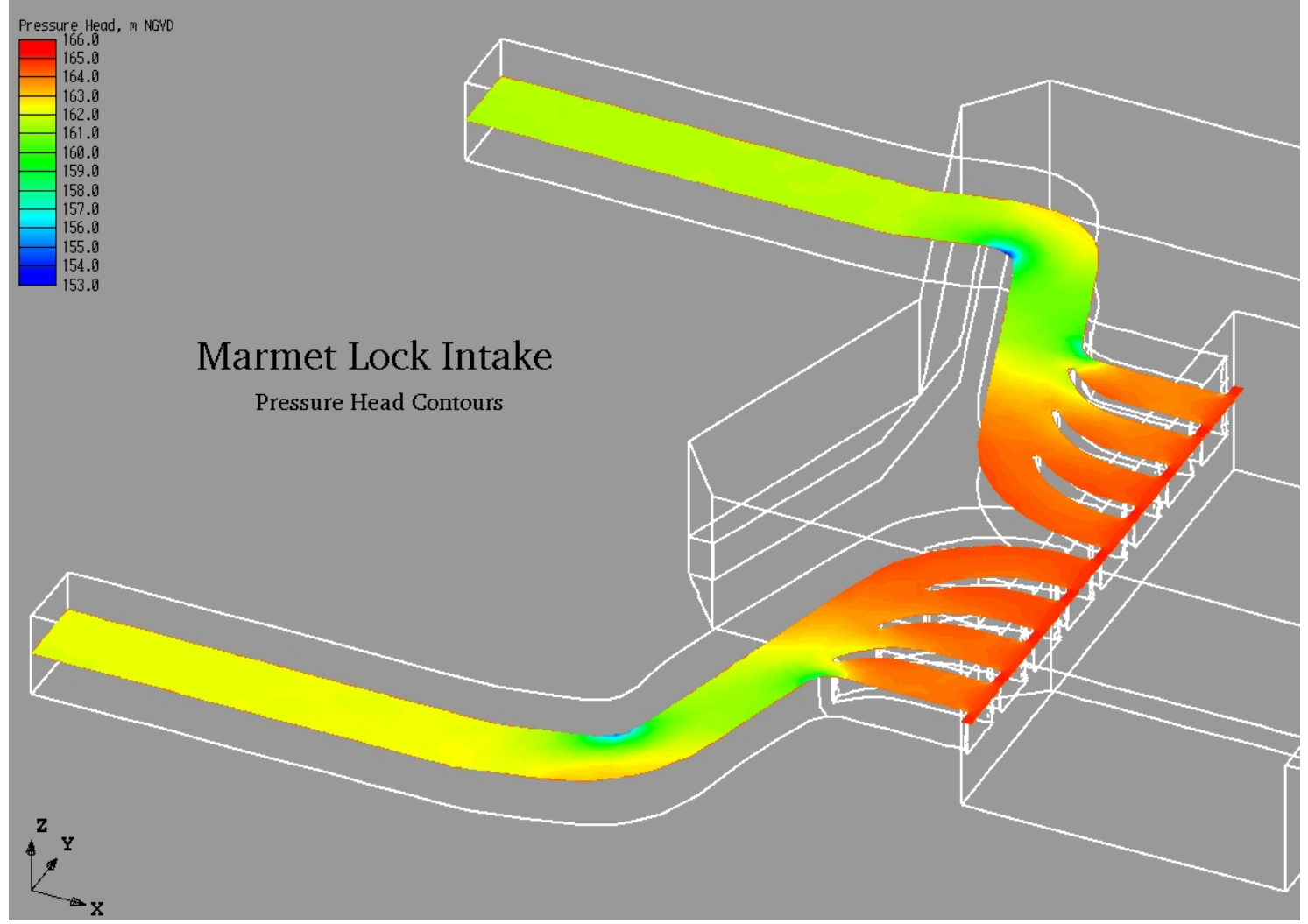

Figure 5. Pressure contours on a plane through center of intake and culverts 


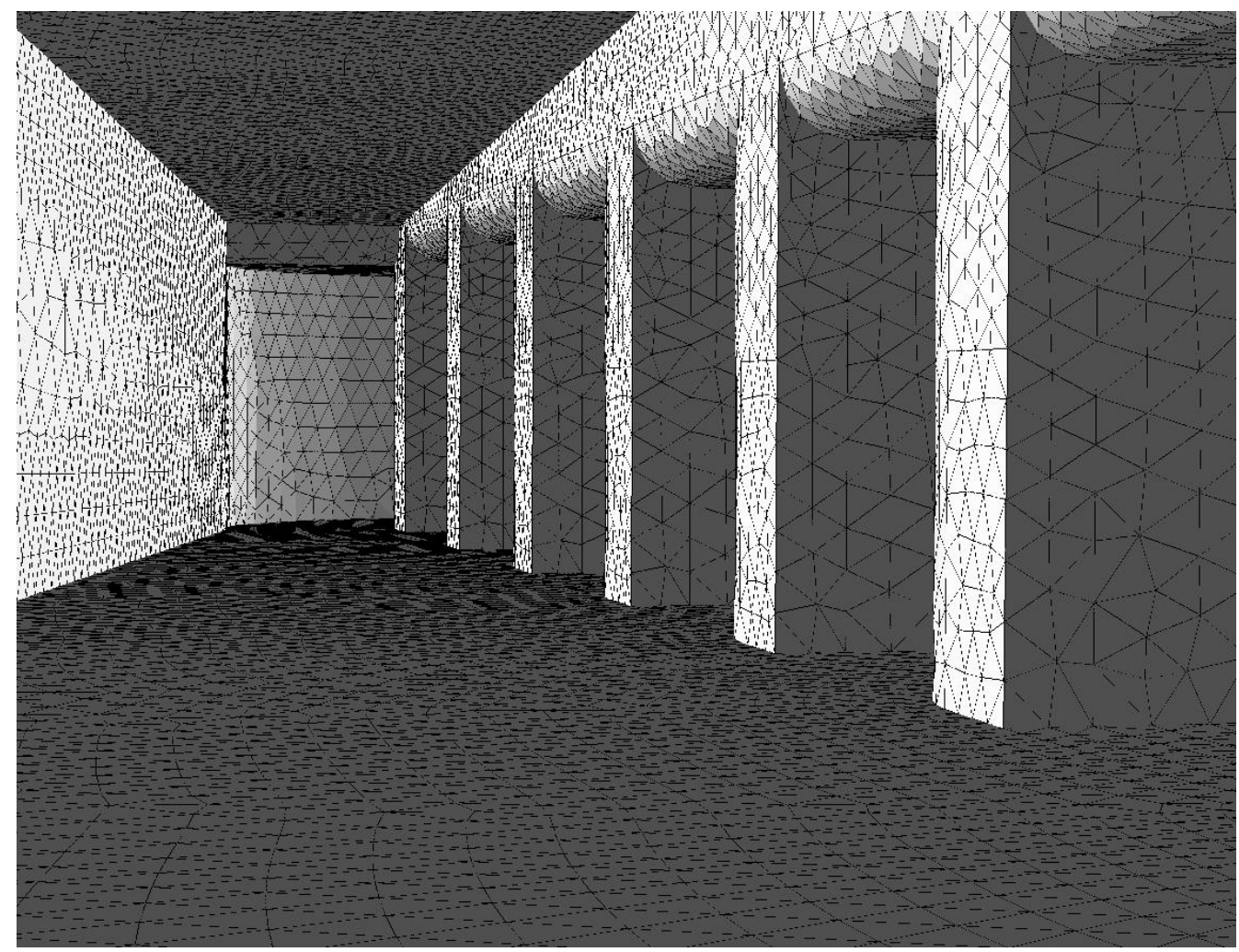

Figure 6. Surface mesh inside multiported outlet

Computational Timings

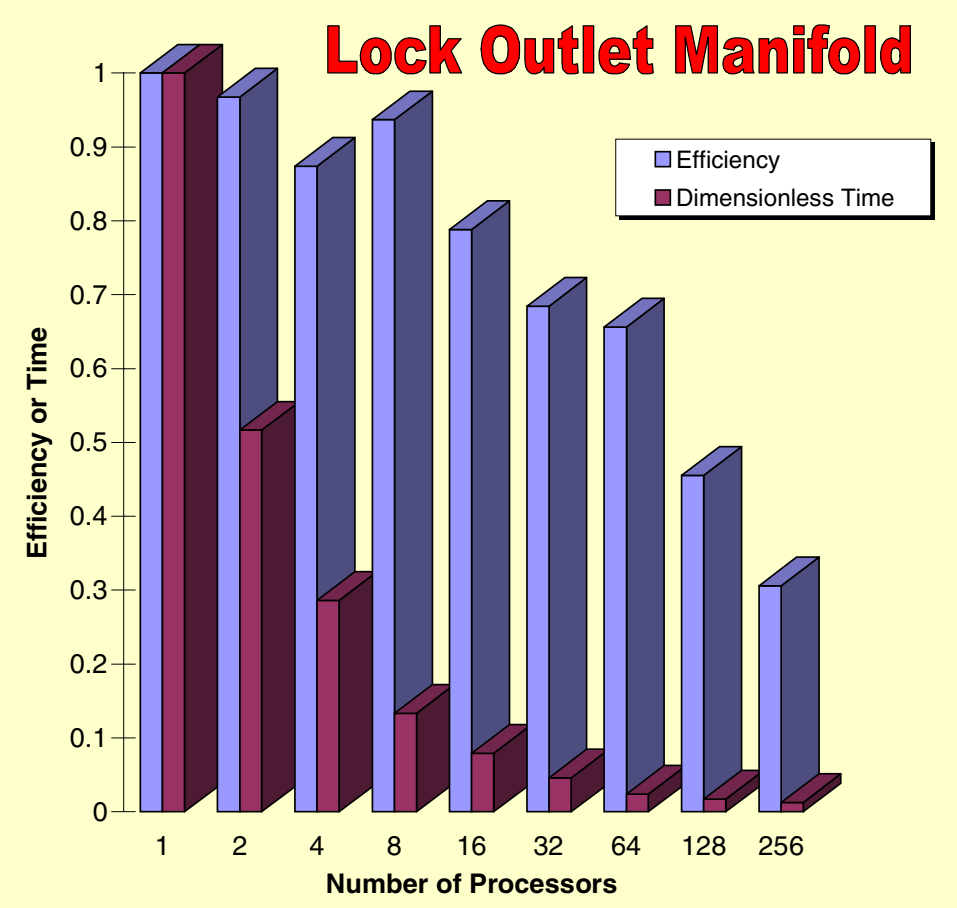

Figure 7. Computational timings for lock outlet manifold model 


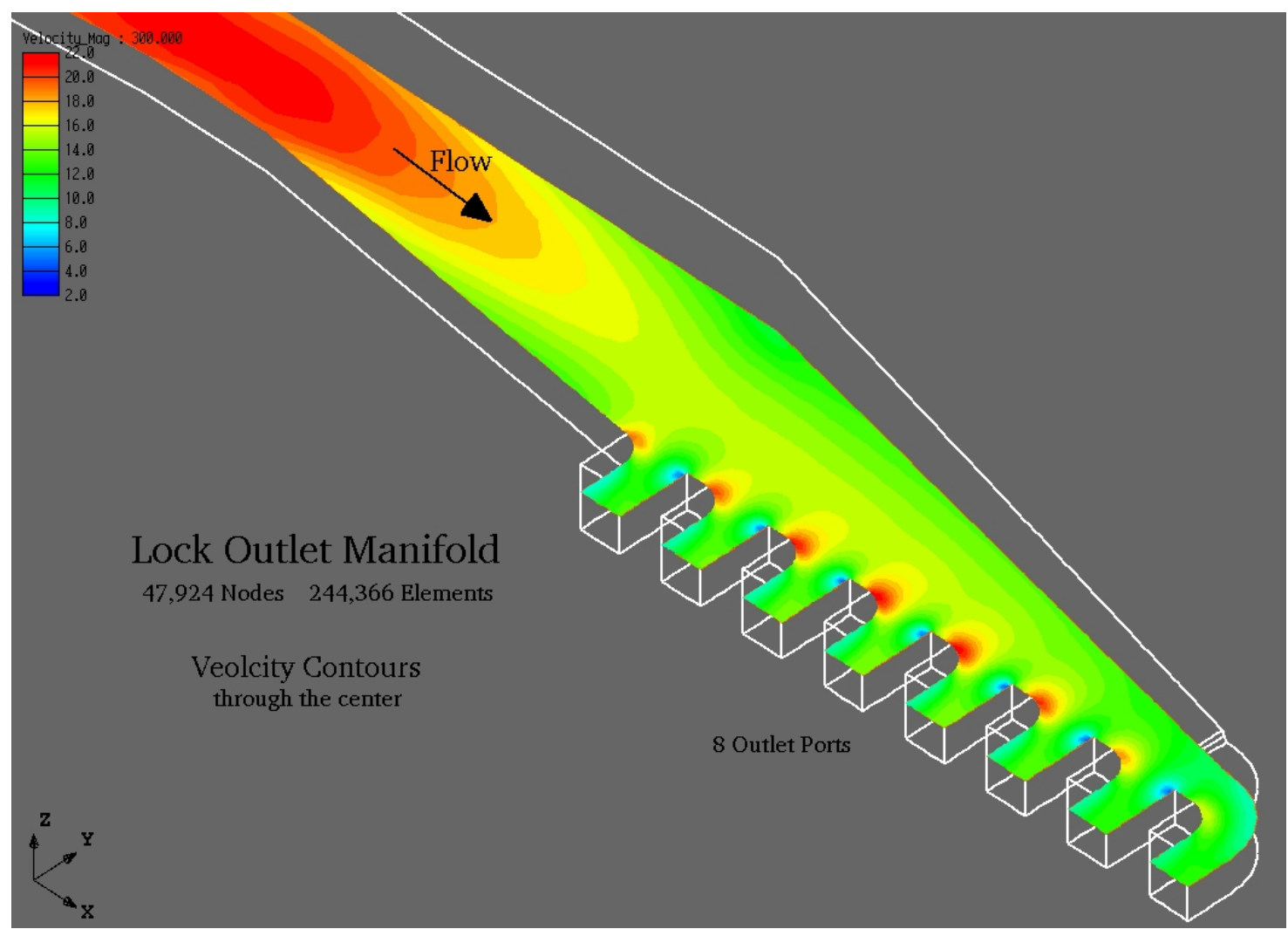

Figure 8. Velocity contours on a plane through center of outlet manifold

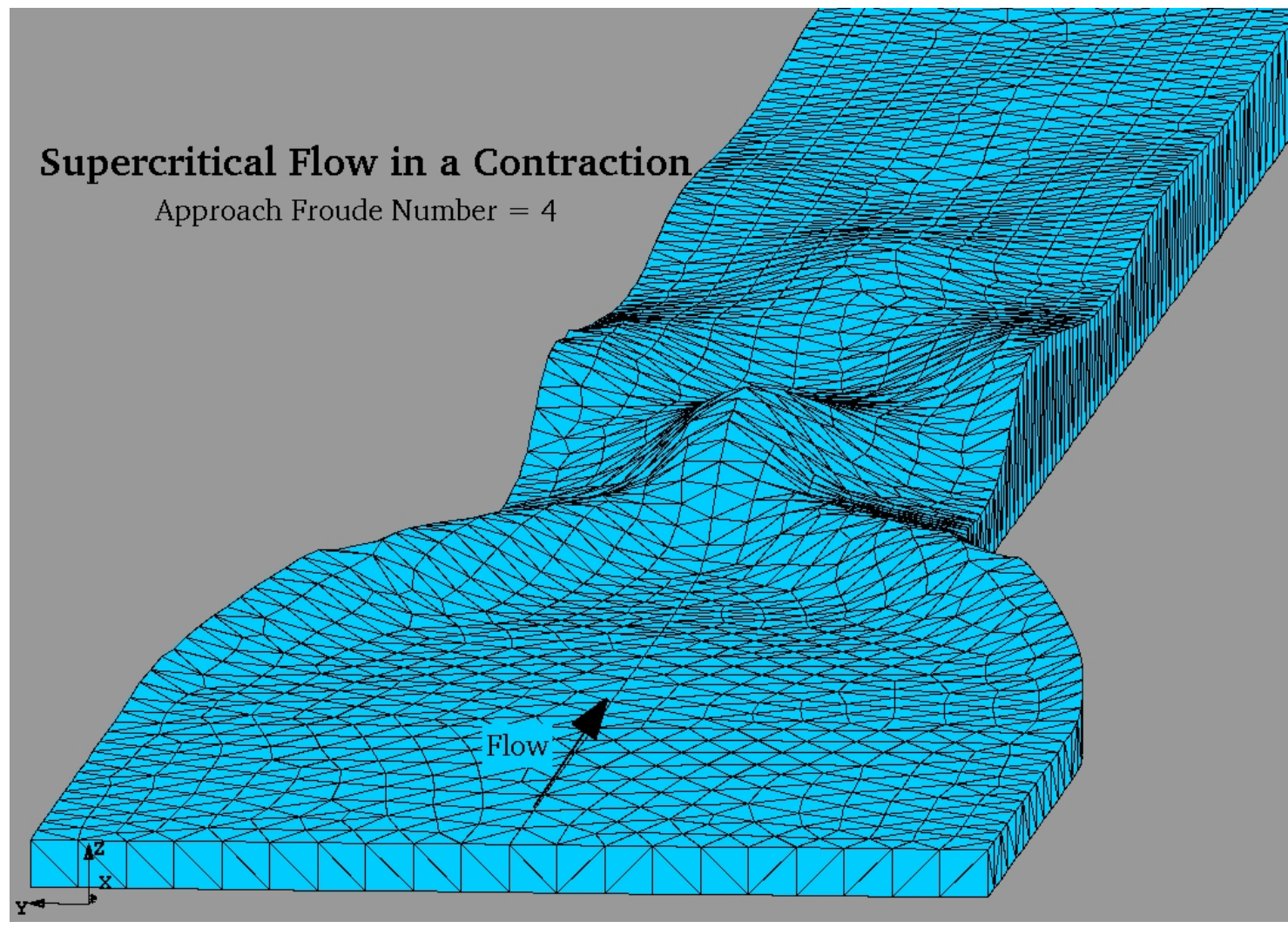

Figure 9. Computational mesh resulting from supercritical flow in a contraction 